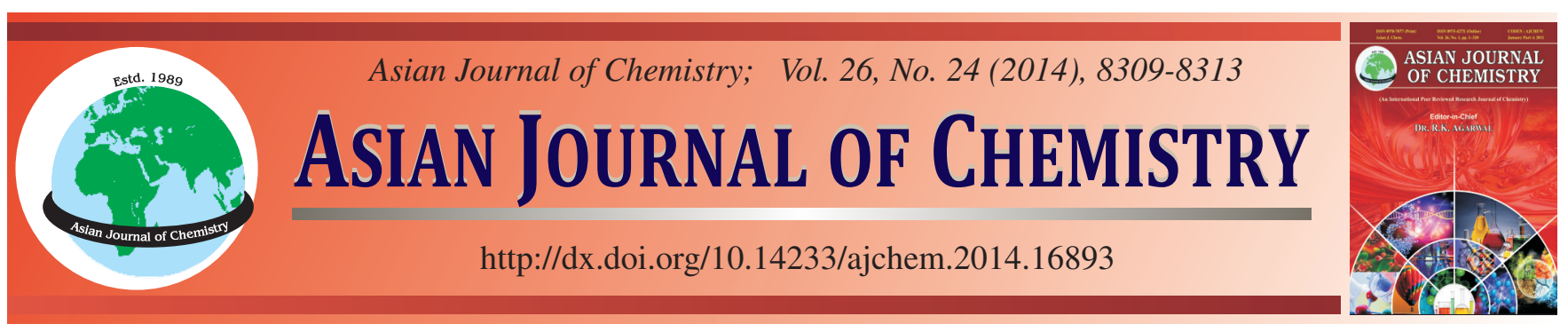

\title{
Synthesis, Characterization and in vitro Antitumor Activity of Novel Schiff Bases Containing Pyrazole Group
}

\author{
Tiegang Ren ${ }^{1}$, Jie WAng ${ }^{1}$, Guinul $\mathrm{LI}^{2, *}$ and Yongzhe $\mathrm{Li}^{1}$
}

${ }^{1}$ Fine Chemistry and Engineering Research Institute, College of Chemistry and Chemical Engineering, Henan University, Kaifeng 475004, P.R. China ${ }^{2}$ Key Laboratory of Ministry of Education for Special Functional Materials, Henan University, Kaifeng 475004, P.R. China

*Corresponding author: Tel: +86 378 2866141; E-mail: rtg@ @enu.edu.cn

\begin{abstract}
A series of novel Schiff base compounds containing pyrazole group were synthesized with pyrazole aldehyde and benzene hydrazine or pyrazole aldehyde and phenylhydrazine hydrochloride as the starting materials. As-synthesized Schiff base compounds were characterized by means of nuclear magnetic resonance spectroscopy, infrared spectrometry, mass spectrometry and elemental analysis. Moreover the in vitro antitumor activity of compounds $\mathbf{B}_{2}$ and $\mathbf{B}_{4}$ against K562 (human leukemia cell line) was evaluated using MTT assay method. Results show that the compounds can inhibit K562 tumor cell's growth and generation.
\end{abstract}

Keywords: Schiff base, Synthesis, Characterize, in vitro Antitumor activity, Heterocyclic compounds, Pyrazole group.

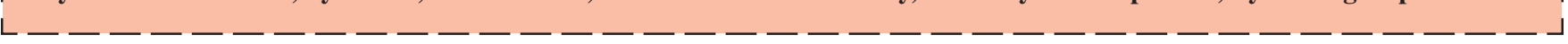

\section{INTRODUCTION}

Schiff base is a kind of organic compounds with special physical and chemical properties ${ }^{1}$. For the strong coordination ability, Schiff base can form complexes with metal or rare earth ions and is a good extractant and chelating agent ${ }^{2-4}$. At the same time, with strong antibacterial and antiviral ability, Schiff base has been widely used in biology, medicine, metallurgy, dye and photoelectric functional materials fields ${ }^{5-8}$. On the other hand, as a kind of efficient extractants and chelators of metal ions ${ }^{9-13}$, pyrazole derivatives have aroused wide concern in biology, medicine, pesticide, analytical applications. Furthermore, the excellent photoelectric properties and carrier transmission capacity of pyrazole derivatives add to their wide use in photoelectric functional materials and organic lightemitting diodes (OLED) ${ }^{14,15}$. Therefore, research of pyrazole based on Schiff base compounds also appears more and more important and valuable. In this article we report a simple procedure for synthesizing Schiff base containing pyrazole group.

\section{EXPERIMENTAL}

Mass spectra (MS) were determined with an Agilent 1100LC-MS mass spectrometer. Infrared (IR) spectra of asobtained products within $4000-400 \mathrm{~cm}^{-1}$ were recorded with a Nicolet 170 SXFT-IR spectrometer (mixed with $\mathrm{KBr}$ and pressed into pellets). Nuclear magnetic resonance $\left({ }^{1} \mathrm{H}\right.$ NMR) spectra in $\left(\mathrm{CD}_{3}\right)_{2} \mathrm{C}=\mathrm{O}$ solvent were recorded with an INOVA-
400 spectrometer in the presence of tetramethylsilane as an internal standard. Elemental analysis was conducted with a PE2400 elemental analysis apparatus.

The in vitro antitumor activity of compounds $\mathbf{B}_{\mathbf{2}}$ and $\mathbf{B}_{\mathbf{4}}$ was evaluated in K562 (human leukemia cell line) using MTT (3-(4,5-dimethylthiazol-2-yl)-2,5-diphenyltetrazolium bromide) assay method (amonafide as reference substance, 10 and $30 \mu \mathrm{mol} / \mathrm{L}$ ). The inhibition rate was calculated from plotted results using untreated cells as $100 \%$.

All chemicals and solvents are of commercial reagent grade and used without further purification. Besides, 1-arylpyrazole4-carbaldehyde was prepared according to procedures available elsewhere. Known structures of as-synthesized products were verified by comparing their data with those reported in the literature ${ }^{16,17}$.

Synthesis of Schiff base compounds: $5 \mathrm{mmol}$ of 1-arylpyrazole-4-carbaldehyde and $5 \mathrm{mmol}$ of benzene hydrazine or phenylhydrazine hydrochloride were completely dissolved in $35 \mathrm{~mL}$ methanol, heated to $80^{\circ} \mathrm{C}$, refluxed for $2 \mathrm{~h}$, cooled to room temperature and placed overnight. After suction filtered and dried, crude mixed products were obtained and purified by recrystallizing in the mixed solvent of $\mathrm{EtOH}$ and $\mathrm{CH}_{2} \mathrm{Cl}_{2}$ $\left(\mathrm{V}_{\mathrm{EtOH}}: \mathrm{V}_{\mathrm{CH}_{2} \mathrm{Cl}_{2}}=1: 3\right)$.

3-Methyl-1-phenyl-4-[(2-(p-tolyl)hydrazono)methyl]$\mathbf{1 H}$-pyrazol-5(4H)-one $\left(\mathbf{A}_{\mathbf{1}}\right)$ : Yellow solid $0.70 \mathrm{~g}$, yield: $46 \%$. APCI-MS $(\mathrm{m} / \mathrm{z})$ calcd. $\left(\mathbf{M}^{-}\right)$306.3, found: 304.9. ${ }^{1} \mathrm{H}$ NMR spectrum (400 MHz, DMSO- $\left.d_{6}\right), \delta$, ppm $(J, H z): 2.19(3 \mathrm{H}, \mathrm{s}$, $\left.\mathrm{CH}_{3}\right), 2.47$ (3H, s, $\left.\mathrm{CH}_{3}\right), 6.89(2 \mathrm{H}, \mathrm{d}, J=8.4, \mathrm{Ar}-\mathrm{H}), 7.02(2 \mathrm{H}$, 
$\mathrm{d}, J=8.2$, Ar-H), $7.46(1 \mathrm{H}, \mathrm{s}, \mathrm{N}=\mathrm{C}-\mathrm{H}), 7.51-7.62(5 \mathrm{H}, \mathrm{m}, \mathrm{Ar}-$ $\mathrm{H}), 7.79(1 \mathrm{H}, \mathrm{s}, \mathrm{N}-\mathrm{H}), 10.22(1 \mathrm{H}, \mathrm{s}, \mathrm{O}-\mathrm{H})$. IR spectrum (thin layer), $\mathrm{v}, \mathrm{cm}^{-1}: 3236(\mathrm{~N}-\mathrm{H}), 1588(\mathrm{C}=\mathrm{N}), 1501,1476(\mathrm{C}=\mathrm{C})$, 1275 (C-N). Anal. calcd.: C, 69.85; H, 5.52; N, 19.17. Found: C, 69.74; H, 5.61; N, $19.13 \%$.

4-[\{2-(4-Fluorophenyl)hydrazono\}methyl]-3-methyl-1phenyl-1H-pyrazol-5(4H)-one $\left(\mathbf{A}_{2}\right)$ : Yellow solid $0.85 \mathrm{~g}$, yield: $55 \%$. APCI-MS $(\mathrm{m} / \mathrm{z})$ calcd. $\left(\mathbf{M}^{-}\right) 310.3$, found: 308.8 . ${ }^{1} \mathrm{H}$ NMR spectrum $\left(400 \mathrm{MHz}, \mathrm{DMSO}-d_{6}\right), \delta$, ppm $(J, \mathrm{~Hz})$ : $2.48\left(3 \mathrm{H}, \mathrm{s}, \mathrm{CH}_{3}\right), 6.93-7.01(2 \mathrm{H}, \mathrm{m}, \mathrm{Ar}-\mathrm{H}), 7.04-7.09(2 \mathrm{H}$, $\mathrm{m}, \mathrm{Ar}-\mathrm{H}), 7.48$ (1H, s, N=C-H), 7.52-7.66 (5H, m, Ar-H), 7.82 $(1 \mathrm{H}, \mathrm{s}, \mathrm{N}-\mathrm{H}), 10.30$ (1H, s, O-H). IR spectrum (thin layer), $\mathrm{v}$, $\mathrm{cm}^{-1}$ : $3244(\mathrm{~N}-\mathrm{H}), 1587(\mathrm{C}=\mathrm{N}), 1503,1477(\mathrm{C}=\mathrm{C}), 1273(\mathrm{C}-\mathrm{N})$. Anal. calcd.: C, 65.80; H, 4.87; N, 18.05. Found: C, 65.87; H, $4.95 ; \mathrm{N}, 18.00 \%$.

4-[\{2-(3-Chlorophenyl)hydrazono\}methyl]-3-methyl1-phenyl-1H-pyrazol-5(4H)-one ( $\left.\mathbf{A}_{3}\right)$ : Yellow solid $0.80 \mathrm{~g}$, yield: $49 \%$. APCI-MS $(\mathrm{m} / \mathrm{z})$ calcd. $\left(\mathrm{M}^{-}\right)$326.7, found: 324.9 . ${ }^{1} \mathrm{H}$ NMR spectrum $\left(400 \mathrm{MHz}, \mathrm{DMSO}-d_{6}\right), \delta$, ppm $(J, \mathrm{~Hz})$ : $2.49\left(\mathrm{~s}, 3 \mathrm{H}, \mathrm{CH}_{3}\right), 6.75(1 \mathrm{H}, \mathrm{dd}, J=7.8,1.2, \mathrm{Ar}-\mathrm{H}), 6.90(1 \mathrm{H}$, $\mathrm{d}, J=8.2, \mathrm{Ar}-\mathrm{H}), 6.99(1 \mathrm{H}, \mathrm{t}, J=1.9, \mathrm{Ar}-\mathrm{H}), 7.23(1 \mathrm{H}, \mathrm{t}, J=$ 8.0, Ar-H), 7.49 (1H, s, N=C-H), 7.53-7.63 (5H, m, Ar-H), $7.86(1 \mathrm{H}, \mathrm{s}, \mathrm{N}-\mathrm{H}), 10.53(1 \mathrm{H}, \mathrm{s}, \mathrm{O}-\mathrm{H})$. IR spectrum (thin layer), $\mathrm{v}, \mathrm{cm}^{-1}: 3234(\mathrm{~N}-\mathrm{H}), 1603(\mathrm{C}=\mathrm{N}), 1502,1479(\mathrm{C}=\mathrm{C}), 1271$ (C-N). Anal. calcd.: C, 62.84; H, 4.63; N, 17.15. Found: C, 62.79; H, 4.71; N, $17.08 \%$.

4-[\{2-(4-Bromophenyl)hydrazono\}methyl]-3-methyl1-phenyl-1H-pyrazol-5(4H)-one $\left(\mathbf{A}_{4}\right)$ : Yellow solid $0.90 \mathrm{~g}$, yield: $48 \%$. APCI-MS ( $\mathrm{m} / \mathrm{z})$ calcd. $\left(\mathbf{M}^{-}\right)$371.2, found: 369.8 . ${ }^{1} \mathrm{H}$ NMR spectrum $\left(400 \mathrm{MHz}, \mathrm{DMSO}-d_{6}\right), \delta$, ppm $(J, \mathrm{~Hz})$ : $2.48\left(3 \mathrm{H}, \mathrm{s}, \mathrm{CH}_{3}\right), 6.91-6.99$ (2H, m, Ar-H), 7.33-7.39 (2H, m, Ar-H), 7.48 (1H, s, N=C-H), 7.53-7.61 (5H, m, Ar-H), 7.84 $(1 \mathrm{H}, \mathrm{s}, \mathrm{N}-\mathrm{H}), 10.46$ (1H, s, O-H). IR spectrum (thin layer), $\mathrm{v}$, $\mathrm{cm}^{-1}$ : $3230(\mathrm{~N}-\mathrm{H}), 1591(\mathrm{C}=\mathrm{N}), 1492,1471(\mathrm{C}=\mathrm{C}), 1278(\mathrm{C}-\mathrm{N})$. Anal. calcd.: C, 55.00; H, 4.07; N, 15.09. Found: C, 54.89; H, 4.13; N, $15.05 \%$.

3-Methyl-4-[\{2-(4-nitrophenyl)hydrazono\}methyl]-1phenyl-1H-pyrazol-5(4H)-one $\left(\mathbf{A}_{5}\right)$ : Deep red solid $0.75 \mathrm{~g}$, yield: $44 \%$. APCI-MS $(\mathrm{m} / \mathrm{z})$ calcd. $\left(\mathrm{M}^{-}\right)$337.3, found: 335.9 . ${ }^{1} \mathrm{H}$ NMR spectrum $\left(400 \mathrm{MHz}, \mathrm{DMSO}-d_{6}\right), \delta$, ppm $(J, \mathrm{~Hz})$ : $2.37\left(3 \mathrm{H}, \mathrm{s}, \mathrm{CH}_{3}\right), 6.98(2 \mathrm{H}, \mathrm{d}, J=8.7, \mathrm{Ar}-\mathrm{H}), 7.17-7.26(1 \mathrm{H}$, m, Ar-H), 7.42 (2H, d, J = 8.2, Ar-H), $7.45(1 \mathrm{H}, \mathrm{s}, \mathrm{N}=\mathrm{C}-\mathrm{H})$, $7.80(2 \mathrm{H}, \mathrm{d}, J=7.8, \mathrm{Ar}-\mathrm{H}), 8.09(2 \mathrm{H}, \mathrm{s}, \mathrm{Ar}-\mathrm{H}), 8.11(1 \mathrm{H}, \mathrm{s}$, $\mathrm{N}-\mathrm{H}), 10.93$ (1H, s, O-H). IR spectrum (thin layer), $\mathrm{v}, \mathrm{cm}^{-1}$ : $3236(\mathrm{~N}-\mathrm{H}), 1596(\mathrm{C}=\mathrm{N}), 1552,1372\left(\mathrm{NO}_{2}\right), 1499,1478$ $(\mathrm{C}=\mathrm{C}), 1277$ (C-N). Anal. calcd.: C, 60.53; H, 4.48; N, 20.76. Found: C, 60.45; H, 4.52; N, $20.71 \%$.

5-Chloro-4-[\{2-(4-fluorophenyl)hydrazono\}methyl]-3methyl-1-phenyl-1H-pyrazole $\left(\mathbf{B}_{1}\right)$ : Deep yellow solid $0.55 \mathrm{~g}$, yield: $33 \%$. APCI-MS $\left(\mathrm{m} / \mathrm{z}\right.$ ) calcd. $\left(\mathrm{M}^{+}\right)$328.7, found: $329.1 .{ }^{1} \mathrm{H}$ NMR spectrum (400 MHz, DMSO- $\left.d_{6}\right), \delta, \operatorname{ppm}(J, \mathrm{~Hz}): 2.60$ $\left(3 \mathrm{H}, \mathrm{s}, \mathrm{CH}_{3}\right), 6.99$ (4H, d, J=6.7, Ar-H), $7.41(1 \mathrm{H}, \mathrm{s}, \mathrm{N}=\mathrm{C}-\mathrm{H})$, 7.46-7.53 (3H, m, Ar-H), 7.54-7.57 (2H, m, Ar-H), $7.70(1 \mathrm{H}, \mathrm{s}$, $\mathrm{N}-\mathrm{H})$. IR spectrum (thin layer), $v, \mathrm{~cm}^{-1}: 3230(\mathrm{~N}-\mathrm{H}), 1597(\mathrm{C}=\mathrm{N})$, 1502, $1478(\mathrm{C}=\mathrm{C}), 1246$ (C-N). Anal. calcd.: C, 62.10; H, 4.29; N, 17.04. Found: C, 62.01; H, 4.37; N, $17.00 \%$.

5-Chloro-4-[\{2-(3-chlorophenyl)hydrazono\}methyl]-3methyl-1-phenyl-1H-pyrazole $\left(\mathbf{B}_{2}\right)$ : Deep yellow solid 1.20 g, yield: $70 \%$. APCI-MS $(\mathrm{m} / \mathrm{z})$ calcd. $\left(\mathrm{M}^{+}\right) 345.2$, found: 346 . ${ }^{1} \mathrm{H}$ NMR spectrum (400 MHz, DMSO- $\left.d_{6}\right), \delta$, ppm $(J, \mathrm{~Hz}): 2.48$ $\left(3 \mathrm{H}, \mathrm{s}, \mathrm{CH}_{3}\right), 6.74(1 \mathrm{H}, \mathrm{dd}, J=7.5,1.6, \mathrm{Ar}-\mathrm{H}), 6.89(1 \mathrm{H}, \mathrm{d}, J=$ 7.4, Ar-H), 6.98 (1H, t, $J=2.0, \mathrm{Ar}-\mathrm{H}), 7.22(1 \mathrm{H}, \mathrm{t}, J=8.1$, ArH), $7.48(1 \mathrm{H}, \mathrm{s}, \mathrm{N}=\mathrm{C}-\mathrm{H}), 7.53-7.60(5 \mathrm{H}, \mathrm{m}, \mathrm{Ar}-\mathrm{H}), 7.85(1 \mathrm{H}, \mathrm{s}$, $\mathrm{N}-\mathrm{H})$. IR spectrum (thin layer), $\mathrm{v}, \mathrm{cm}^{-1}: 3234(\mathrm{~N}-\mathrm{H}), 1598(\mathrm{C}=\mathrm{N})$, 1501, $1474(\mathrm{C}=\mathrm{C}), 1246(\mathrm{C}-\mathrm{N})$. Anal. calcd.: C, 59.14; H, 4.09; N, 16.23. Found: C, 59.10; H, 4.14; N, $16.20 \%$.

4-[\{2-(4-Bromophenyl)hydrazono\}methyl]-5-chloro-3methyl-1-phenyl-1 $\boldsymbol{H}$-pyrazole $\left(\mathbf{B}_{3}\right)$ : Deep yellow solid 1.10 g, yield: $56 \%$. APCI-MS $(\mathrm{m} / \mathrm{z})$ calcd. $(\mathrm{M}+\mathrm{Na})^{+} 389.6$, found: 412.1. ${ }^{1} \mathrm{H}$ NMR spectrum (400 MHz, DMSO- $d_{6}$ ), $\delta$, ppm $(J, \mathrm{~Hz}): 2.60\left(3 \mathrm{H}, \mathrm{s}, \mathrm{CH}_{3}\right), 6.94(2 \mathrm{H}, \mathrm{d}, J=7.5, \mathrm{Ar}-\mathrm{H}), 7.36$ $(2 \mathrm{H}, \mathrm{d}, J=8.7, \mathrm{Ar}-\mathrm{H}), 7.41(1 \mathrm{H}, \mathrm{s}, \mathrm{N}=\mathrm{C}-\mathrm{H}), 7.47-7.52(3 \mathrm{H}, \mathrm{m}$, Ar-H), 7.54-7.57 (2H, m, Ar-H), 7.70 (1H, s, N-H). IR spectrum (thin layer), $\mathrm{v}, \mathrm{cm}^{-1}: 3236(\mathrm{~N}-\mathrm{H}), 1596(\mathrm{C}=\mathrm{N}), 1500,1470$ $(\mathrm{C}=\mathrm{C}), 1253$ (C-N). Anal. calcd.: C, 52.40; H, 3.62; N, 14.38 . Found: C, 52.31; H, 3.71; N, $14.37 \%$.

5-Chloro-3-methyl-4-[\{2-(4-nitrophenyl)hydrazono\}methyl]-1-phenyl-1H-pyrazole (B $\left.\mathbf{B}_{4}\right)$ : Brick red solid $1.35 \mathrm{~g}$, yield: $76 \%$. APCI-MS $(\mathrm{m} / \mathrm{z})$ calcd. $\left(\mathrm{M}^{+}\right)$355.7, found: 356.2 . ${ }^{1} \mathrm{H}$ NMR spectrum (400 MHz, DMSO- $\left.d_{6}\right), \delta$, ppm $(J, \mathrm{~Hz})$ : $2.50\left(3 \mathrm{H}, \mathrm{s}, \mathrm{CH}_{3}\right), 7.08(2 \mathrm{H}, \mathrm{d}, J=7.3, \mathrm{Ar}-\mathrm{H}), 7.50(1 \mathrm{H}, \mathrm{s}$, $\mathrm{N}=\mathrm{C}-\mathrm{H}), 7.54-7.61$ (5H, m, Ar-H), 8.04 (1H, s, N-H), 8.13 $(2 \mathrm{H}, \mathrm{d}, J=8.8, \mathrm{Ar}-\mathrm{H})$. IR spectrum (thin layer), $\mathrm{v}, \mathrm{cm}^{-1}: 3237$ $(\mathrm{N}-\mathrm{H}), 1601(\mathrm{C}=\mathrm{N}), 1554,1382\left(\mathrm{NO}_{2}\right), 1501,1476(\mathrm{C}=\mathrm{C})$, 1241 (C-N). Anal. calcd.: C, 57.39; H,3.97; N, 19.68. Found: C, $57.30 ; \mathrm{H}, 4.01 ; \mathrm{N}, 19.65 \%$.

3,5-Dimethyl-1-phenyl-4-[(2-phenylhydrazono)methyl]$\mathbf{H} \boldsymbol{H}$-pyrazole $\left(\mathbf{C}_{1}\right)$ : Deep yellow solid $0.50 \mathrm{~g}$, yield: $34 \%$. APCI-MS $(\mathrm{m} / \mathrm{z})$ calcd. $\left(\mathbf{M}^{+}\right)$290.3, found: 291.2. ${ }^{1} \mathrm{H}$ NMR spectrum (400 MHz, DMSO- $\left.d_{6}\right), \delta$, ppm $(J, \mathrm{~Hz}): 2.38(3 \mathrm{H}, \mathrm{s}$, $\left.\mathrm{CH}_{3}\right), 2.43\left(3 \mathrm{H}, \mathrm{s}, \mathrm{CH}_{3}\right), 6.68(1 \mathrm{H}, \mathrm{t}, J=7.3, \mathrm{Ar}-\mathrm{H}), 6.96(2 \mathrm{H}$, $\mathrm{d}, J=7.7, \mathrm{Ar}-\mathrm{H}), 7.18(2 \mathrm{H}, \mathrm{t}, J=7.8, \mathrm{Ar}-\mathrm{H}), 7.41(1 \mathrm{H}, \mathrm{s}, \mathrm{N}=\mathrm{C}-$ H), 7.51-7.54 (5H, m, Ar-H), $7.90(1 \mathrm{H}, \mathrm{s}, \mathrm{N}-\mathrm{H})$. IR spectrum (thin layer), $\mathrm{v}, \mathrm{cm}^{-1}$ : $3236(\mathrm{~N}-\mathrm{H}), 1600(\mathrm{C}=\mathrm{N}), 1504,1428$ $(\mathrm{C}=\mathrm{C}), 1263(\mathrm{C}-\mathrm{N})$. Anal. calcd.: C, 74.45; H, 6.25; N, 19.30. Found: C, 74.39; H, 6.18; N, $19.27 \%$.

4-[\{2-(4-Fluorophenyl)hydrazono $\}$ methyl $]-3,5-$ dimethyl-1-phenyl-1H -pyrazole $\left(\mathbf{C}_{2}\right)$ : Deep yellow solid 0.75 g, yield: $49 \%$. APCI-MS ( $m / z$ ) calcd. $\left(\mathbf{M}^{+}\right)$308.3, found: 309.1. ${ }^{1} \mathrm{H}$ NMR spectrum (400 MHz, DMSO- $d_{6}$ ), $\delta$, ppm $(J, \mathrm{~Hz}): 2.37\left(3 \mathrm{H}, \mathrm{s}, \mathrm{CH}_{3}\right), 2.42\left(3 \mathrm{H}, \mathrm{s}, \mathrm{CH}_{3}\right), 6.93(2 \mathrm{H}, \mathrm{dd}, J=$ 9, 4.8, Ar-H), 7.03 (2H, t, $J=8.9$, Ar-H), $7.40(1 \mathrm{H}, \mathrm{s}, \mathrm{N}=\mathrm{C}-$ H), 7.46-7.56 (5H, m, Ar-H), $7.89(1 \mathrm{H}, \mathrm{s}, \mathrm{N}-\mathrm{H})$. IR spectrum (thin layer), $\mathrm{v}, \mathrm{cm}^{-1}$ : $3222(\mathrm{~N}-\mathrm{H}), 1596(\mathrm{C}=\mathrm{N}), 1502,1425$ $(\mathrm{C}=\mathrm{C}), 1272(\mathrm{C}-\mathrm{N})$. Anal. calcd.: $\mathrm{C}, 70.11 ; \mathrm{H}, 5.56$; N, 18.17 . Found: C, 70.05; H, 5.63; N, $18.01 \%$.

4-[\{2-(3-Chlorophenyl)hydrazono $\}$ methyl $]-3,5-$ dimethyl-1-phenyl-1H-pyrazole $\left(\mathbf{C}_{3}\right)$ : Deep yellow solid 0.55 g, yield: $34 \%$. APCI-MS $\left(\mathrm{m} / \mathrm{z}\right.$ ) calcd. $\left(\mathrm{M}^{+}\right)$324.8, found: 325.1. ${ }^{1} \mathrm{H}$ NMR spectrum (400 MHz, DMSO- $\left.d_{6}\right), \delta$, ppm $(J$, $\mathrm{Hz}): 2.37\left(3 \mathrm{H}, \mathrm{s}, \mathrm{CH}_{3}\right), 2.43\left(3 \mathrm{H}, \mathrm{s}, \mathrm{CH}_{3}\right), 6.69(1 \mathrm{H}, \mathrm{s}, \mathrm{Ar}-\mathrm{H})$, $6.86(1 \mathrm{H}, \mathrm{s}, \mathrm{Ar}-\mathrm{H}), 6.95(1 \mathrm{H}, \mathrm{s}, \mathrm{Ar}-\mathrm{H}), 7.19(1 \mathrm{H}, \mathrm{s}, \mathrm{Ar}-\mathrm{H})$, $7.42(1 \mathrm{H}, \mathrm{s}, \mathrm{N}=\mathrm{C}-\mathrm{H}), 7.48-7.52(5 \mathrm{H}, \mathrm{m}, \mathrm{Ar}-\mathrm{H}), 7.91(1 \mathrm{H}, \mathrm{s}$, $\mathrm{N}-\mathrm{H})$. IR spectrum (thin layer), $\mathrm{v}, \mathrm{cm}^{-1}: 3224(\mathrm{~N}-\mathrm{H}), 1597$ $(\mathrm{C}=\mathrm{N}), 1504,1430(\mathrm{C}=\mathrm{C}), 1265(\mathrm{C}-\mathrm{N})$. Anal. calcd.: C, 66.56; H, 5.28; N, 17.25. Found: C, 66.50; H, 5.29; N, $17.21 \%$. 
4-[\{2-(4-Bromophenyl)hydrazono\}methyl $]-3,5$ dimethyl-1-phenyl-1 $\boldsymbol{H}$-pyrazole $\left(\mathbf{C}_{\mathbf{4}}\right)$ : Deep yellow solid 1.05 g, yield: $57 \%$. APCI-MS $(\mathrm{m} / \mathrm{z})$ calcd. $\left(\mathrm{M}^{+}\right)$369.2, found: 370.1 . ${ }^{1} \mathrm{H}$ NMR spectrum $\left(400 \mathrm{MHz}, \mathrm{DMSO}-d_{6}\right), \delta$, ppm $(J, \mathrm{~Hz})$ : $2.37\left(3 \mathrm{H}, \mathrm{s}, \mathrm{CH}_{3}\right), 2.42\left(3 \mathrm{H}, \mathrm{s}, \mathrm{CH}_{3}\right), 6.90(2 \mathrm{H}, \mathrm{t}, J=5.9$, Ar$\mathrm{H}), 7.32(2 \mathrm{H}, \mathrm{t}, J=5.9$, Ar-H), $7.41(1 \mathrm{H}, \mathrm{s}, \mathrm{N}=\mathrm{C}-\mathrm{H}), 7.48-$ 7.54 (5H, m, Ar-H), 7.90 (1H, s, N-H). IR spectrum (thin layer), $\mathrm{v}, \mathrm{cm}^{-1}: 3236(\mathrm{~N}-\mathrm{H}), 1596(\mathrm{C}=\mathrm{N}), 1500,1427(\mathrm{C}=\mathrm{C}), 1266$ (C-N). Anal. calcd.: C, 58.55; H, 4.64; N, 15.17. Found: C, 58.50; H,4.71; N, $15.12 \%$.

3,5-Dimethyl-4-[\{2-(4-nitrophenyl)hydrazono\}methyl]1-phenyl-1H-pyrazole $\left(\mathbf{C}_{5}\right)$ : Brick red solid 1.40 g, yield: $83 \%$. APCI-MS $(\mathrm{m} / \mathrm{z})$ calcd. $\left(\mathrm{M}^{+}\right)$335.3, found: 336.1. ${ }^{1} \mathrm{H}$ NMR spectrum (400 MHz, DMSO- $\left.d_{6}\right), \delta, \operatorname{ppm}(J, \mathrm{~Hz}): 2.40$ $\left(3 \mathrm{H}, \mathrm{s}, \mathrm{CH}_{3}\right), 2.45$ (3H, s, $\left.\mathrm{CH}_{3}\right), 7.04(2 \mathrm{H}, \mathrm{s}, \mathrm{Ar}-\mathrm{H}), 7.42(1 \mathrm{H}$, s, C=N-H), 7.48-7.59 (5H, m, Ar-H), $8.10(2 \mathrm{H}, \mathrm{s}, \mathrm{Ar}-\mathrm{H}), 8.12$ $(1 \mathrm{H}, \mathrm{s}, \mathrm{N}-\mathrm{H})$. IR spectrum (thin layer), $\mathrm{v}, \mathrm{cm}^{-1}: 3231(\mathrm{~N}-\mathrm{H})$, $1595(\mathrm{C}=\mathrm{N}), 1553,1378\left(\mathrm{NO}_{2}\right), 1503,1432(\mathrm{C}=\mathrm{C}), 1262$ (C-N). Anal. calcd.: C, 64.47; H, 5.11; N, 20.88. Found: C, $64.45 ; \mathrm{H}, 5.10 ; \mathrm{N}, 20.78 \%$.

1-(4-Bromophenyl)-3,5-dimethyl-4-[(2-phenyl hydrazono)methyl]-1H-pyrazole ( $\left.\mathbf{D}_{1}\right)$ : Yellow solid $1.55 \mathrm{~g}$, yield: $84 \%$. APCI-MS $(\mathrm{m} / \mathrm{z})$ calcd. $\left(\mathrm{M}^{+}\right)$369.3, found: $370.1 .{ }^{1} \mathrm{H}$ NMR spectrum (400 MHz, DMSO- $\left.d_{6}\right), \delta, \operatorname{ppm}(J, \mathrm{~Hz}): 2.48$ $\left(3 \mathrm{H}, \mathrm{s}, \mathrm{CH}_{3}\right), 2.49\left(3 \mathrm{H}, \mathrm{s}, \mathrm{CH}_{3}\right), 6.85(1 \mathrm{H}, \mathrm{s}, \mathrm{Ar}-\mathrm{H}), 7.04(3 \mathrm{H}$, s, Ar-H), 7.31-7.35 (3H, m, Ar-H), 7.58-7.60 (2H, m, Ar-H), $7.61(1 \mathrm{H}, \mathrm{s}, \mathrm{N}=\mathrm{C}-\mathrm{H}), 7.75(1 \mathrm{H}, \mathrm{s}, \mathrm{N}-\mathrm{H})$. IR spectrum (thin layer), $\mathrm{v}, \mathrm{cm}^{-1}$ : $3231(\mathrm{~N}-\mathrm{H}), 1600(\mathrm{C}=\mathrm{N}), 1496,1425(\mathrm{C}=\mathrm{C})$, 1255 (C-N). Anal. calcd.: C, 58.55; H, 4.64; N, 15.17. Found: C, 58.51; H, 4.69; N, $15.07 \%$.

1-(4-Bromophenyl)-3,5-dimethyl-4-[\{2-(p-tolyl) hydrazono\}methyl]-1H -pyrazole $\left(\mathbf{D}_{\mathbf{2}}\right)$ : Yellow solid $1.45 \mathrm{~g}$, yield: $76 \%$. APCI-MS $(\mathrm{m} / \mathrm{z})$ calcd. $\left(\mathrm{M}^{+}\right)$383.3, found: 384.0 . ${ }^{1} \mathrm{H}$ NMR spectrum $\left(400 \mathrm{MHz}, \mathrm{DMSO}-d_{6}\right), \delta$, ppm $(J, \mathrm{~Hz})$ : $2.18\left(3 \mathrm{H}, \mathrm{s}, \mathrm{CH}_{3}\right), 2.36\left(3 \mathrm{H}, \mathrm{s}, \mathrm{CH}_{3}\right), 2.43\left(3 \mathrm{H}, \mathrm{s}, \mathrm{CH}_{3}\right), 6.85$ $(2 \mathrm{H}, \mathrm{d}, J=8.4, \mathrm{Ar}-\mathrm{H}), 6.98(2 \mathrm{H}, \mathrm{t}, J=9.1, \mathrm{Ar}-\mathrm{H}), 7.47-7.50$ $(2 \mathrm{H}, \mathrm{m}, \mathrm{Ar}-\mathrm{H}), 7.69$ (1H, s, N=C-H), 7.70-7.77 (2H, m, Ar$\mathrm{H}), 7.84$ (1H, s, N-H). IR spectrum (thin layer), $\mathrm{v}, \mathrm{cm}^{-1}: 3235$ $(\mathrm{N}-\mathrm{H}), 1607(\mathrm{C}=\mathrm{N}), 1498,1426(\mathrm{C}=\mathrm{C}), 1254(\mathrm{C}-\mathrm{N})$. Anal. calcd.: C, 49.54; H, 5; N, 14.62. Found: C, 49.46; H, 5.07; N, $14.67 \%$.

1-(4-Bromophenyl)-4-[\{2-(4-fluorophenyl)hydrazono\}methyl]-3,5-dimethyl-1H-pyrazole $\left(\mathbf{D}_{3}\right)$ : Yellow solid 1.30 g, yield: $67 \%$. APCI-MS $(\mathrm{m} / \mathrm{z})$ calcd. $\left(\mathrm{M}^{+}\right)$387.3, found: 388.1 . ${ }^{1} \mathrm{H}$ NMR spectrum (400 MHz, DMSO- $\left.d_{6}\right), \delta$, ppm $(J, \mathrm{~Hz})$ : $2.36\left(3 \mathrm{H}, \mathrm{s}, \mathrm{CH}_{3}\right), 2.43\left(3 \mathrm{H}, \mathrm{s}, \mathrm{CH}_{3}\right), 6.88-6.98(2 \mathrm{H}, \mathrm{m}, \mathrm{Ar}-\mathrm{H})$, $7.02(2 \mathrm{H}, \mathrm{m}, \mathrm{Ar}-\mathrm{H}), 7.48(2 \mathrm{H}, \mathrm{t}, J=7.9, \mathrm{Ar}-\mathrm{H}), 7.69(1 \mathrm{H}, \mathrm{s}$, $\mathrm{N}=\mathrm{C}-\mathrm{H}), 7.70-7.77$ (2H, m, Ar-H), 7.88 (1H, s, N-H). IR spectrum (thin layer), $\mathrm{v}, \mathrm{cm}^{-1}: 3233(\mathrm{~N}-\mathrm{H}), 1606(\mathrm{C}=\mathrm{N}), 1500$, $1426(\mathrm{C}=\mathrm{C}), 1253(\mathrm{C}-\mathrm{N})$. Anal. calcd.: C, 55.83; H, 4.16; N, 14.47. Found: C, 55.79; H, 4.19; N, $14.39 \%$.

1-(4-Bromophenyl)-4-[\{2-(4-bromophenyl)hydrazono\}methyl]-3,5-dimethyl-1H-pyrazole $\left(\mathbf{D}_{4}\right)$ : Yellow solid 1.50 g, yield: $67 \%$. APCI-MS $(\mathrm{m} / \mathrm{z})$ calcd. $(\mathrm{M}+\mathrm{Na})^{+} 448.2$, found: 471.8. ${ }^{1} \mathrm{H}$ NMR spectrum (400 MHz, DMSO- $\left.d_{6}\right), \delta$, ppm $(J$, $\mathrm{Hz}): 2.36\left(3 \mathrm{H}, \mathrm{s}, \mathrm{CH}_{3}\right), 2.42\left(3 \mathrm{H}, \mathrm{s}, \mathrm{CH}_{3}\right), 6.84-6.98(2 \mathrm{H}, \mathrm{m}$, Ar-H), 7.26-7.41 (2H, m, Ar-H), 7.45-7.57 (2H, m, Ar-H), $7.69(1 \mathrm{H}, \mathrm{s}, \mathrm{N}=\mathrm{C}-\mathrm{H}), 7.71-7.75(2 \mathrm{H}, \mathrm{m}, \mathrm{Ar}-\mathrm{H}), 7.88(1 \mathrm{H}, \mathrm{s}$, $\mathrm{N}-\mathrm{H})$. IR spectrum (thin layer), $\mathrm{v}, \mathrm{cm}^{-1}: 3236(\mathrm{~N}-\mathrm{H}), 1594$
$(\mathrm{C}=\mathrm{N}), 1496,1426(\mathrm{C}=\mathrm{C}), 1252(\mathrm{C}-\mathrm{N})$. Anal. calcd.: C, 48.24; H, 3.60; N, 12.50. Found: C, 48.20; H, 3.69; N, $12.47 \%$.

1-(4-Bromophenyl)-3,5-dimethyl-4-[\{2-(4-nitrophenyl)hydrazono\}methyl]-1H-pyrazole $\left(\mathbf{D}_{5}\right)$ : Brick red solid 1.90 g, yield: $92 \%$. APCI-MS $(\mathrm{m} / \mathrm{z})$ calcd. $(\mathrm{M}+\mathrm{Na})^{+} 437.3$, found: 437.1. ${ }^{1} \mathrm{H}$ NMR spectrum (400 MHz, DMSO- $\left.d_{6}\right), \delta$, ppm $(J$, $\mathrm{Hz}): 2.49\left(3 \mathrm{H}, \mathrm{s}, \mathrm{CH}_{3}\right), 2.51\left(3 \mathrm{H}, \mathrm{s}, \mathrm{CH}_{3}\right), 7.01(2 \mathrm{H}, \mathrm{t}, J=7.2$, Ar-H), 7.31-7.35 (2H, m, Ar-H), 7.60-7.64 (2H, m, Ar-H), $7.84(1 \mathrm{H}, \mathrm{s}, \mathrm{N}=\mathrm{C}-\mathrm{H}), 7.91(1 \mathrm{H}, \mathrm{s}, \mathrm{N}-\mathrm{H}), 8.81(2 \mathrm{H}, \mathrm{d}, J=9.3$, Ar-H). IR spectrum (thin layer), $\mathrm{v}, \mathrm{cm}^{-1}: 3231(\mathrm{~N}-\mathrm{H}), 1606$ $(\mathrm{C}=\mathrm{N})$, 1553, $1378\left(\mathrm{NO}_{2}\right), 1499,1427(\mathrm{C}=\mathrm{C}), 1254(\mathrm{C}-\mathrm{N})$. Anal. calcd.: C, 52.19; H, 3.89; N, 16.91. Found: C, 52.11; H, $3.99 ; \mathrm{N}, 16.84 \%$.

3,5-Dimethyl-1-(4-nitrophenyl)-4-[(2-phenyl hydrazono)methyl]-1H-pyrazole $\left(\mathbf{E}_{\mathbf{1}}\right)$ : Deep red solid $1.10 \mathrm{~g}$, yield: $66 \%$. APCI-MS $(\mathrm{m} / \mathrm{z})$ calcd. $\left(\mathrm{M}^{+}\right)$335.4, found: 336.7. ${ }^{1} \mathrm{H}$ NMR spectrum (400 MHz, DMSO-d $)$, $\delta, \operatorname{ppm}(J, \mathrm{~Hz}): 2.42$ $\left(3 \mathrm{H}, \mathrm{s}, \mathrm{CH}_{3}\right), 2.55\left(3 \mathrm{H}, \mathrm{s}, \mathrm{CH}_{3}\right), 6.69(1 \mathrm{H}, \mathrm{t}, J=7.3, \mathrm{Ar}-\mathrm{H})$, 6.96 (2H, d, J = 7.6, Ar-H), 7.12-7.29 (2H, m, Ar-H), 7.837.87 (2H, m, Ar-H), 7.89 (1H, s, N=C-H), 8.30-8.41 (2H, m, Ar-H), 10.11 (1H, s, N-H). IR spectrum (thin layer), $\mathrm{v}, \mathrm{cm}^{-1}$ : $3235(\mathrm{~N}-\mathrm{H}), 1600(\mathrm{C}=\mathrm{N}), 1551,1376\left(\mathrm{NO}_{2}\right), 1503,1427$ $(\mathrm{C}=\mathrm{C}), 1262(\mathrm{C}-\mathrm{N})$. Anal. calcd.: C, 64.47; H, 5.11; N, 20.88. Found: C, 64.38; H, 5.18; N, $20.82 \%$.

3,5-Dimethyl-1-(4-nitrophenyl)-4-[\{2-(p-tolyl)hydrazono\}methyl]-1H -pyrazole $\left(\mathbf{E}_{\mathbf{2}}\right)$ : Deep red solid 1.30 g, yield: $74 \%$. APCI-MS $(\mathrm{m} / \mathrm{z})$ calcd. $\left(\mathrm{M}^{+}\right)$349.4, found: 350.2. ${ }^{1} \mathrm{H}$ NMR spectrum (400 MHz, DMSO- $\left.d_{6}\right), \delta, \operatorname{ppm}(J, \mathrm{~Hz}): 2.19$ $\left(3 \mathrm{H}, \mathrm{s}, \mathrm{CH}_{3}\right), 2.40\left(3 \mathrm{H}, \mathrm{s}, \mathrm{CH}_{3}\right), 2.55\left(3 \mathrm{H}, \mathrm{s}, \mathrm{CH}_{3}\right), 6.87(2 \mathrm{H}$, $\mathrm{d}, J=8.4, \mathrm{Ar}-\mathrm{H}), 7.00(2 \mathrm{H}, \mathrm{d}, J=8.2, \mathrm{Ar}-\mathrm{H}), 7.83(1 \mathrm{H}, \mathrm{s}$, $\mathrm{N}=\mathrm{C}-\mathrm{H}), 7.85-7.89$ (2H, m, Ar-H), 8.31-8.39 (2H, m, Ar-H), $9.98(1 \mathrm{H}, \mathrm{s}, \mathrm{N}-\mathrm{H})$. IR spectrum (thin layer), $\mathrm{v}, \mathrm{cm}^{-1}: 3236$ $(\mathrm{N}-\mathrm{H}), 1595(\mathrm{C}=\mathrm{N}), 1550,1380\left(\mathrm{NO}_{2}\right), 1506,1428(\mathrm{C}=\mathrm{C})$, 1260 (C-N). Anal. calcd.: C, 65.32; H, 5.48; N, 20.04. Found: C, 65.25; H, 5.51; N, $20.07 \%$.

4-[\{2-(4-Fluorophenyl)hydrazono $\}$ methyl] -3,5dimethyl-1-(4-nitrophenyl)-1H-pyrazole $\left(\mathbf{E}_{3}\right)$ : Deep red solid $1.35 \mathrm{~g}$, yield: $76 \%$. APCI-MS $(\mathrm{m} / \mathrm{z})$ calcd. $\left(\mathbf{M}^{+}\right)$353.4, found: 354.2. ${ }^{1} \mathrm{H}$ NMR spectrum (400 MHz, DMSO- $d_{6}$ ), $\delta$, $\operatorname{ppm}(\mathrm{J}, \mathrm{Hz}): 2.40\left(3 \mathrm{H}, \mathrm{s}, \mathrm{CH}_{3}\right), 2.54\left(3 \mathrm{H}, \mathrm{s}, \mathrm{CH}_{3}\right), 6.92-6.97$ (2H, m, Ar-H,), 7.00-7.09 (2H, m, Ar-H), 7.81-7.86 (2H, m, Ar-H), 7.87 (1H, s, N=C-H), 8.30-8.40 (2H, m, Ar-H), 10.09 $(1 \mathrm{H}, \mathrm{s}, \mathrm{N}-\mathrm{H})$. IR spectrum (thin layer), $\mathrm{v}, \mathrm{cm}^{-1}: 3235(\mathrm{~N}-\mathrm{H})$, $1594(\mathrm{C}=\mathrm{N}), 1553,1370\left(\mathrm{NO}_{2}\right), 1504,1430(\mathrm{C}=\mathrm{C}), 1267(\mathrm{C}-$ N). Anal. calcd.: C, 61.18; H, 4.56; N, 19.82. Found: C, 61.11; H, 4.59; N, $19.77 \%$.

4-[\{2-(3-Chlorophenyl)hydrazono\}methyl]-3,5-dimethyl1-(4-nitrophenyl)-1 $\boldsymbol{H}$-pyrazole $\left(\mathbf{E}_{\mathbf{4}}\right)$ : Deep red solid $1.25 \mathrm{~g}$, yield: $68 \%$. APCI-MS $(\mathrm{m} / \mathrm{z})$ calcd. $\left(\mathrm{M}^{+}\right) 369.8$, found: 370.1 . ${ }^{1} \mathrm{H}$ NMR spectrum $\left(400 \mathrm{MHz}, \mathrm{DMSO}-d_{6}\right), \delta$, ppm $(J, \mathrm{~Hz})$ : $2.40\left(3 \mathrm{H}, \mathrm{s}, \mathrm{CH}_{3}\right), 2.54$ (3H, s, $\left.\mathrm{CH}_{3}\right), 6.88-6.98(2 \mathrm{H}, \mathrm{m}, \mathrm{Ar}-\mathrm{H})$, 7.00-7.10 (2H, m, Ar-H), 7.81-7.86 (2H, m, Ar-H), $7.87(1 \mathrm{H}$, s, N=C-H), 8.30-8.39 (2H, m, Ar-H), 10.09 (1H, s, N-H). IR spectrum (thin layer), $\mathrm{v}, \mathrm{cm}^{-1}: 3234(\mathrm{~N}-\mathrm{H}), 1594(\mathrm{C}=\mathrm{N}), 1556$, $1386\left(\mathrm{NO}_{2}\right), 1506,1429(\mathrm{C}=\mathrm{C}), 1268(\mathrm{C}-\mathrm{N})$. Anal. calcd.: C, 58.46; H, 4.36; N, 18.94. Found: C, 58.45; H, 4.39; N, $18.91 \%$.

4-[\{2-(4-Bromophenyl)hydrazono\}methyl]-3,5-dimethyl1-(4-nitrophenyl)-1H-pyrazole $\left(\mathbf{E}_{5}\right)$ : Deep red solid $1.25 \mathrm{~g}$, yield: $60 \%$. APCI-MS ( $\mathrm{m} / \mathrm{z}$ ) calcd. $\left(\mathrm{M}^{+}\right) 414.5$, found: 415.3 . 
${ }^{1} \mathrm{H}$ NMR spectrum $\left(400 \mathrm{MHz}, \mathrm{DMSO}-d_{6}\right), \delta$, ppm $(J, \mathrm{~Hz})$ : $2.50\left(3 \mathrm{H}, \mathrm{s}, \mathrm{CH}_{3}\right), 2.60\left(3 \mathrm{H}, \mathrm{s}, \mathrm{CH}_{3}\right), 6.93-7.00(2 \mathrm{H}, \mathrm{m}, \mathrm{Ar}-\mathrm{H})$, 7.35-7.37 (2H, m, Ar-H), 7.67-7.70 (2H, m, Ar-H), $7.73(1 \mathrm{H}$, s, N=C-H), 8.35-8.38 (2H, m, Ar-H), 10.05 (1H, s, N-H). IR spectrum (thin layer), $\mathrm{v}, \mathrm{cm}^{-1}: 3231(\mathrm{~N}-\mathrm{H}), 1601(\mathrm{C}=\mathrm{N}), 1553$, $1378\left(\mathrm{NO}_{2}\right), 1503,1427(\mathrm{C}=\mathrm{C}), 1263(\mathrm{C}-\mathrm{N})$. Anal. calcd.: C, 52.19; H, 3.89; N, 16.91. Found: C, 52.13; H, 3.97; N, $16.88 \%$.

3,5-Dimethyl-1-(4-nitrophenyl)-4-[\{2-(4-nitrophenyl)hydrazono\}methyl]-1H-pyrazole $\left(\mathbf{E}_{\mathbf{6}}\right)$ : Brick red solid 1.40 g, yield: $74 \%$. APCI-MS $(\mathrm{m} / \mathrm{z})$ calcd. $\left(\mathrm{M}^{+}\right)$380.4, found: 381.5 . ${ }^{1} \mathrm{H}$ NMR spectrum $\left(400 \mathrm{MHz}, \mathrm{DMSO}-d_{6}\right), \delta$, ppm $(J, \mathrm{~Hz})$ : $2.44\left(3 \mathrm{H}, \mathrm{s}, \mathrm{CH}_{3}\right), 2.57\left(3 \mathrm{H}, \mathrm{s}, \mathrm{CH}_{3}\right), 7.05(2 \mathrm{H}, \mathrm{d}, J=8, \mathrm{Ar}-\mathrm{H})$, 7.82-7.93 (2H, m, Ar-H), 8.10-8.11 (2H, m, Ar-H), $8.13(1 \mathrm{H}$, s, N=C-H), 8.30-8.45 (2H, m, Ar-H), 11.26 (1H, s, N-H). IR spectrum (thin layer), $\mathrm{v}, \mathrm{cm}^{-1}: 3236(\mathrm{~N}-\mathrm{H}), 1596(\mathrm{C}=\mathrm{N}), 1556$, $1387\left(\mathrm{NO}_{2}\right), 1502,1427(\mathrm{C}=\mathrm{C}), 1266(\mathrm{C}-\mathrm{N})$. Anal. calcd.: C, 56.84; H, 4.24; N, 22.10. Found: C, 56.80; H, 4.29; N, $22.02 \%$.

\section{RESULTS AND DISCUSSION}

These Schiff base compounds were synthesized by condensation reaction with 1-arylpyrazol-4-carbaldehyde and benzene hydrazine or phenylhydrazine hydrochloride as the starting materials (Fig.1). The phenylhydrazine hydrochloride can dissociate by weak base (e.g., $\mathrm{CH}_{3} \mathrm{COONa}$ ), but it was found that the post processing of the reaction would become very complex after adding $\mathrm{CH}_{3} \mathrm{COONa}$ and the products were difficult to separate out from the reaction system. Generally, weak acid can be used as a catalyst in this reaction, however, it was found that it is disadvantageous to the separation of products as adding acid and phenylhydrazine hydrochloride as the substrate maybe enhance the acidity of reaction system, so there is no other acid has been added as catalyst. In the meantime, the results showed that we can get good yields and pure products in the presence of methanol as the solvent and refluxing $2 \mathrm{~h}$ under $80{ }^{\circ} \mathrm{C}$. It is relatively complex to the purification of Schiff base compounds, for the Schiff base can decompose in the silica gel column and these compounds can't be separated by column layer chromatography. At last, the crystallization method has been used to separate these Schiff base compounds while EtOH and $\mathrm{CH}_{2} \mathrm{Cl}_{2}\left(\mathrm{~V}_{\mathrm{EtOH}}: \mathrm{V}_{\mathrm{CH}_{2} \mathrm{Cl}_{2}}=1: 3\right)$ as solvent.
The existence of electron-withdrawing substituent on the pyrazole ring is beneficial to the reaction and the experimental results show that the reaction is easier and the yield of the target compounds is higher when $\mathrm{R}_{1}=\mathrm{Cl}$ and $\mathrm{R}_{3}=\mathrm{F}, \mathrm{Cl}, \mathrm{Br}$ and $-\mathrm{NO}_{2}$ as compared with the electron-donating substituent on the pyrazole ring. For $\mathrm{R}_{2}$ the existence of electron-donating substituent is disadvantageous to the reaction, but it's easier for product to separate out from the reaction system when $\mathrm{R}_{2}=-\mathrm{NO}_{2}$.

Structural characterization: The structure of target compounds have been characterized by means of ${ }^{1} \mathrm{H}$ NMR, FT-IR, MS and elemental analysis. In the ${ }^{1} \mathrm{H}$ NMR spectrum, it was found that the $\mathrm{N}=\mathrm{C}-\mathrm{H}$ chemical shift was at 7.40-8.13 (7.45-7.49 for A-series; 7.40-7.50 for B-series; 7.40-7.42 for C-series; 7.61-7.84 for D-series; 7.73-8.13 for E-series), is a singlet; it can be seen that the data clearly are divided into two groups and have small difference for it, the results illustrate that $\mathrm{R}_{3}$ has certain influence to the chemical shift of $\mathrm{N}=\mathrm{C}-\mathrm{H}$ but these influence is very less. For A-series the chemical shift of $\mathrm{O}-\mathrm{H}$ is located in the scope of 10.22 to 10.93 with a strong singlet. The N-H appeared in the range of 7.69 to 8.12 (7.798.11 for A- series; 7.69-8.04 for B-series; 7.87-8.12 for Cseries; 7.75-7.91 for D-series) and 9.98-11.26 for E-series. The abnormal of E-series can be interpreted as the influence of the intermolecular hydrogen bond. In the IR spectrum, the absorption of N-H peak was within the scope of 3237-3222 $\mathrm{cm}^{-1}$; The stretching vibration absorption of $\mathrm{C}=\mathrm{N}$ appeared in the range of $1607-1587 \mathrm{~cm}^{-1} ; 1506-1425 \mathrm{~cm}^{-1}$ was the scope of absorption peaks of aromatic ring skeleton; $1278-1241 \mathrm{~cm}^{-1}$ belonged to the vibration absorption peak of C-N. And it appeared two obviously characteristic absorption peaks, in 1556-1550 and 1382-1370 $\mathrm{cm}^{-1}$, respectively for the compounds that containing the substituent of $-\mathrm{NO}_{2}$.

in vitro Antitumor activity: We predict that the as-synthesis Schiff base compounds containing pyrazole group has biological activity for the Schiff base and pyrazole derivatives have been widely used in biomedical fields. For this reason, we choose compounds $\mathrm{B}_{2}$ and $\mathrm{B}_{4}$ as examples to explore their in vitro antitumor activity. Their antiproliferative activity against K562 (human leukemia cell line) was evaluated by in vitro MTT assays. Amonafide was tested as a reference compound. The biological results were shown in Table-1. It can be seen<smiles>[R4]c1cccc(N/N=C/c2c(C)nn(-c3cccc([R19])c3)c2[R])c1</smiles>

$$
\begin{aligned}
& \mathrm{R}_{1}=\mathrm{OH} ; \mathrm{R}_{3}=\mathrm{H} ; \mathrm{R}_{2}=p-\mathrm{CH}_{3}\left(\mathbf{A}_{\mathbf{1}}\right) ; p-\mathrm{F}\left(\mathbf{A}_{\mathbf{2}}\right) ; m-\mathrm{Cl}\left(\mathbf{A}_{\mathbf{3}}\right) ; p-\mathrm{Br}\left(\mathbf{A}_{\mathbf{4}}\right) ; p-\mathrm{NO}_{2}\left(\mathbf{A}_{\mathbf{5}}\right) \\
& \mathrm{R}_{1}=\mathrm{Cl} ; \mathrm{R}_{3}=\mathrm{H} ; \mathrm{R}_{2}=p-\mathrm{F}\left(\mathbf{B}_{1}\right) ; m-\mathrm{Cl}\left(\mathbf{B}_{2}\right) ; p-\mathrm{Br}\left(\mathbf{B}_{3}\right) ; p-\mathrm{NO}_{2}\left(\mathbf{B}_{\mathbf{4}}\right) \\
& \mathrm{R}_{1}=\mathrm{CH}_{3} ; \mathrm{R}_{3}=\mathrm{H} ; \mathrm{R}_{2}=\mathrm{H}\left(\mathbf{C}_{\mathbf{1}}\right) ; p-\mathrm{F}\left(\mathbf{C}_{2}\right) ; m-\mathrm{Cl}\left(\mathbf{C}_{\mathbf{3}}\right) ; p-\mathrm{Br}\left(\mathbf{C}_{\mathbf{4}}\right) ; p-\mathrm{NO}_{2}\left(\mathbf{C}_{\mathbf{5}}\right) \\
& \mathrm{R}_{1}=\mathrm{CH}_{3} ; \mathrm{R}_{3}=p-\mathrm{Br} ; \mathrm{R}_{2}=\mathrm{H}\left(\mathbf{D}_{\mathbf{1}}\right) ; p-\mathrm{CH}_{3}\left(\mathbf{D}_{2}\right) ; p-\mathrm{F}\left(\mathbf{D}_{\mathbf{3}}\right) ; p-\mathrm{Br}\left(\mathbf{D}_{\mathbf{4}}\right) ; p-\mathrm{NO}_{2}\left(\mathbf{D}_{\mathbf{5}}\right) \\
& \mathrm{R}_{1}=\mathrm{CH}_{3} ; \mathrm{R}_{3}=p-\mathrm{NO}_{2} ; \mathrm{R}_{2}=\mathrm{H}\left(\mathbf{E}_{\mathbf{1}}\right) ; p-\mathrm{CH}_{3}\left(\mathbf{E}_{\mathbf{2}}\right) ; p-\mathrm{F}\left(\mathbf{E}_{\mathbf{3}}\right) ; m-\mathrm{Cl}\left(\mathbf{E}_{\mathbf{4}}\right) ; p-\mathrm{Br}\left(\mathbf{E}_{\mathbf{5}}\right) ; p-\mathrm{NO}_{2}\left(\mathbf{E}_{\mathbf{6}}\right)
\end{aligned}
$$

Fig. 1. Synthesis of Schiff base compounds 
from Table-1 that the compounds $\mathbf{B}_{2}$ and $\mathbf{B}_{4}$ have showed antiproliferative activity against K562 and can inhibit the growth of K562 cells.

\begin{tabular}{ccc}
\multicolumn{3}{c}{ TABLE-1 } \\
\multicolumn{2}{c}{ INHIBITION RATES OF COMPOUNDS } \\
$\mathbf{B}_{\mathbf{2}}$ AND $\mathbf{B}_{\mathbf{4}}$ TO K562 CELL LINES \\
\hline \multirow{2}{*}{ Compound } & \multicolumn{2}{c}{ K562 (\%) } \\
\cline { 2 - 3 } & $10 \mu \mathrm{mol} \mathrm{L}^{-1}$ & $30 \mu \mathrm{mol} \mathrm{L}^{-1}$ \\
\hline Amonafide & 18.5 & 39.0 \\
$\mathbf{B}_{2}$ & 10.6 & 11.8 \\
$\mathbf{B}_{4}$ & 0.31 & 9.1 \\
\hline
\end{tabular}

\section{Conclusion}

We have synthesized 5 series novel Schiff bases containing pyrazole group and characterized by means of ${ }^{1} \mathrm{H}$ NMR, FTIR, MS and elemental analysis. The test shows that the compounds $\mathbf{B}_{\mathbf{2}}$ and $\mathbf{B}_{\mathbf{4}}$ have showed antiproliferative activity against K562 and can inhibit the growth of K562 cells.

\section{REFERENCES}

1. S. Yamada, Coord. Chem. Rev., 190-192, 537 (1999).

2. K. Mekouar, J.F. Mouscadet, D. Desmaële, F. Subra, H. Leh, D. Savouré, C. Auclair and J. d'Angelo, J. Med. Chem., 41, 2846 (1998).
3. R.N. Jadeja, J.R. Shah, E. Suresh and P. Paul, Polyhedron, 23, 2465 (2004).

4. M.B.R. Bastos, J.C. Moreira and P.A.M. Farias, Anal. Chim. Acta, 408, 83 (2000).

5. S. Sadeghi, F. Fathi, A.A. Esmaeili and H. Naeimi, Sens. Actuators B, 114, 928 (2006).

6. S.B. Desai, P.B. Desai and K.R. Desai, Heterocycl. Commun., 7, 83 (2001).

7. S. Banthia and A. Samanta, Phys. Chem. B, 110, 6437 (2006).

8. S.J. Toal, K.A. Jones, D. Magde and W.C. Trogler, J. Am. Chem. Soc., 127, 11661 (2005).

9. R.I. Kureshy, N.H. Khan, S.H.R. Abdi, S.T. Patel and R.V. Jasra, Tetrahedron Lett., 42, 2915 (2001).

10. C.A. Sureshan and P.K. Bhattacharya, J. Mol. Catal. Chem., 136, 285 (1998).

11. A. Tong, Y. Akama and S. Tanaka, J. Chromatogr. A, 478, 408 (1989).

12. Y.H. Liu, X.L. Liu and D.M. Yuan, Chin. J. Org. Chem., 25, 893 (2005).

13. Z. Cimerman, N. Galic and B. Bosner, Anal. Chim. Acta, 343, 145 (1997).

14. F.R. Pérez, J. Belmar, Y. Moreno, R. Baggio and O. Peña, New J. Chem., 29, 283 (2005).

15. F. Marchetti, C. Pettinari, R. Pettinari, A. Cingolani, D. Leonesi and A. Lorenzotti, Polyhedron, 18, 3041 (1999).

16. J. Guo, T.G. Ren, J.L. Zhang, G.H. Li, W.J. Li and L.R. Yang, Spectrochim. Acta A, 95, 135 (2012).

17. T.G. Ren, H.B. Cheng, J.L. Zhang, W.J. Li, J. Guo and L.R. Yang, J. Fluoresc., 22, 201 (2012). 